\title{
Cardiovascular Adverse Events Reported from COVID-19 Vaccines: A Study Based on WHO Database
}

\author{
Rimple Jeet Kaur (D) \\ Siddhartha Dutta (D) \\ Jaykaran Charan (D) \\ Pankaj Bhardwaj (D) ${ }^{2}$ \\ Ankita Tandon (iD ${ }^{3}$ \\ Dharamveer Yadav (iD) 4 \\ Salequl Islam iD ${ }^{5}$ \\ Mainul Haque (iD) ${ }^{6}$ \\ 'Department of Pharmacology, All India \\ Institute of Medical Sciences, Jodhpur, \\ Rajasthan, India; ${ }^{2}$ Department of \\ Community and Family Medicine, All \\ India Institute of Medical Sciences \\ (AlIMS), Jodhpur, Rajasthan, India; \\ ${ }^{3}$ Department of Oral Pathology, \\ Microbiology and Forensic Odontology, \\ Dental Institute, Rajendra Institute of \\ Medical Sciences, Ranchi, 834009, India; \\ ${ }^{4}$ Department of Biochemistry, All India \\ Institute of Medical Sciences, Jodhpur, \\ Rajasthan, India; ${ }^{5}$ Department of \\ Microbiology, Jahangirnagar University, \\ Savar, Dhaka, 1342, Bangladesh; 'Unit of \\ Pharmacology, Faculty of Medicine and \\ Defence Health, Universiti Pertahanan \\ Nasional Malaysia (National Defence \\ University of Malaysia), Kem Perdana \\ Sungai Besi, 57000, Kuala Lumpur, \\ Malaysia
}

Correspondence: Jaykaran Charan Department of Pharmacology, All India Institute of Medical Sciences, Jodhpur, Rajasthan, India

Email dr.jaykaran78@gmail.com

Mainul Haque

Unit of Pharmacology, Faculty of Medicine and Defence Health, Universiti

Pertahanan Nasional Malaysia (National

Defence University of Malaysia), Kem

Perdana Sungai Besi, 57000, Kuala

Lumpur, Malaysia

Email runurono@gmail.com
Background: Thirteen COVID-19 vaccines are granted emergency approval. It is crucial to monitor their adverse events post vaccination. The present study focuses on cardiovascular adverse events post-COVID-19 vaccination and aims to determine adverse events with the administered vaccine.

Methodology: The cardiovascular (CVS) adverse events were extracted for three broad headings (SOCs) - cardiac disorders, vascular disorders, and investigations. Descriptive statistics were reported in the form of percentage and frequency, and the disproportionality analysis was conducted.

Results: For the cardiovascular system, 4863 adverse events (AEs) were reported from BNT162b2 Pfizer, 1222 AstraZeneca, Moderna, and other COVID-19 vaccines. Common adverse events observed with vaccines under study were tachycardia (16.41\%), flushing (12.17\%), hypertension (5.82\%), hypotension (3.60\%) and peripheral coldness $(2.41 \%)$. Based on disproportionality analysis ( $\mathrm{IC}_{025}$ values), acute myocardial infarction, cardiac arrest, and circulatory collapse were linked to the vaccines in the age group $>75$ years. Hypertension, severe hypertension, supraventricular tachycardia, sinus tachycardia, and palpitations were associated across all age groups and either gender. Amongst the investigations, abnormal ECG findings raised C-reactive protein, elevated D dimer, and troponin were reported in specific age groups or gender or all subjects.

Conclusion: Although cardiovascular events have been reported with the COVID-19 vaccines, the causality is yet to be established because such CVS AEs are also usually associated with the general public even without intervention. Hence, people should be administered these vaccines, and sustained monitoring of these AEs should be done.

Keywords: adverse events, COVID-19 vaccines, cardiovascular adverse events, Moderna, BNT162b2, 1222 AstraZeneca

\section{Introduction}

COVID-19, a SARS-CoV-2 RNA virus-associated acute respiratory illness, emerged in December 2019 in Wuhan, China, and was transmitted globally precipitously. Since its origin, as of 24th March 2021, it has infected 123,419,065 individuals and led to about $2,719,163$ deaths. ${ }^{1}$ For over a year, scientists across the globe have faced complex challenges to combat the disease. Research for COVID-19 treatment included repurposing existing drugs along with the discovery of new drugs and vaccines. Currently, about thirteen COVID-19 vaccines are granted emergency approval in various countries, and include Comirnaty (BNT162b2), Moderna COVID-19 Vaccine (mRNA- 
1273), COVID-19 Vaccine AstraZeneca (AZD1222); also known as Covishield, Sputnik V, COVID-19 Vaccine Janssen (JNJ-78436735; Ad26.COV2.S), CoronaVac, BBIBP-CorV, EpiVacCorona, Convidicea (Ad5-nCoV), Covaxin, CoviVac, ZF2001, no name announced (China). ${ }^{2}$ Due to an urgent need to prevent the further spread of COVID-19 infections, the conventional procedures for approval of new drug/vaccines could not be followed, and these vaccines were given emergency approval before completion of all three phases of clinical trials. This generates a solid reason for accumulating sound scientific evidence for these vaccines by monitoring the adverse events in the population post-vaccination.

Since during the study period, three vaccines, Comirnaty (BNT162b2), Moderna COVID-19 Vaccine (mRNA-1273), COVID-19 Vaccine AstraZeneca (AZD1222), were widely used for vaccination; therefore, analysis from the adverse events reported in VigiBase was performed on these vaccines. Pfizer and Biotech developed the BNT162b2 vaccine; it is a lipid nanoparticle-formulated, nucleoside-modified RNA vaccine that encodes a prefusion stabilized, membrane-anchored SARS-CoV-2 full-length spike protein. ${ }^{3}$ The mRNA-1273 is a lipid-nanoparticle (LNP)-encapsulated mRNA vaccine expressing the prefusion-stabilized spike glycoprotein, was developed by Moderna and the Vaccine Research Center at the National Institute of Allergy and Infectious Diseases (NIAID), within the National Institutes of Health (NIH). ${ }^{4}$ The ChAdOx1 nCoV-19 vaccine AZD1222 (ChAdOx1 nCoV-19 vaccine) was developed at Oxford University and consists of a replication-deficient chimpanzee adenoviral vector ChAdOx1, containing the SARS-CoV-2 structural surface glycoprotein antigen (spike protein; $\mathrm{nCoV}-19)$ gene. ${ }^{5}$

At present, there is scattered information regarding the adverse events without a concrete database with a compiled information about the reporting /analysis of adverse events following the COVID-19 vaccination. ${ }^{6-10}$ The Centers for Disease Control and Prevention (CDC) analyzed COVID-19 adverse events and reported the same on the Vaccine Adverse Event Reporting System (VAERS) of the United States. This suggested that most of the adverse events reported for COVID-19 vaccines in the US were mild and had very few serious adverse events. ${ }^{11}$

VigiBase is an international pharmacovigilance post-marketing database of the World Health Organization, which contains adverse events of drugs reported worldwide. ${ }^{12}$ Literature shows that VigiBase was previously used to analyze the adverse events related to several repurposed drugs for COVID-19. ${ }^{13-16}$ In the absence of definite post-vaccination robust data on adverse events related to the COVID-19 vaccines, most of the studies conducted on COVID-19 vaccines are based on the data generated from these vaccines' clinical trials and on the data available in the VigiBase. ${ }^{17-19}$ Since all COVID-19 vaccines are new drugs; hence it is essential to monitor their adverse events post-approval. Thus, we analyzed all the cardiovascular adverse events reported with COVID-19 vaccination in the WHO global pharmacovigilance database VigiBase and assessed any relationship between the adverse events with the vaccine. This study is also intended to generate a safety signal for COVID19 vaccines at an early stage and form the basis of other studies generating or evaluating the safety data of COVID-19 vaccines.

\section{Methodology}

We used VigiBase for this study, a global pharmacovigilance database maintained by World Health Organization. The VigiBase was established in 1968 and consisted of over 20 million reports of adverse events reported by the WHO program's member countries for International Drug Monitoring in VigiBase. ${ }^{12}$ The adverse events are reported in individual case safety reports (ICSRs) by health professionals. It is a repository of ICSR of adverse events collected by the national pharmacovigilance centers of about 130 member countries. ${ }^{12,20,21}$ The adverse events reported in the VigiBase are organized in a structured format and include information regarding the patient (age, gender, country, continent of residence), drugs (start and end date, route of administration, and indication of use), adverse event details (date of onset, seriousness, causality, and outcome) and the administrative details (type and source of report). In this database, the medicines are coded following the WHO Drug Dictionary enhanced including the Anatomical Therapeutic Classification (ATC), and the adverse events are coded as per WHO adverse reaction Terminology and the Medical Dictionary for Regulatory Authorities (MedDRA). ${ }^{22,23}$ MedDRA contains precise standardized medical terminology to facilitate the global sharing of uniform regulatory information for medical products used by humans. ${ }^{24}$ As per MedDRA, the information in VigiBase is arranged in a particular hierarchical order with five levels: LTTs (Lowest Level Terms), PTs (Preferred Terms), HLTs (High-Level Terms), HLGTs (High-Level Group Terms), and SOCs (System Organ Classes). ${ }^{25}$ 
The data for adverse events related to COVID-19 vaccines was obtained on a subscription basis from VigiBase. We used SOC (System Organ Class) information and PT (Preferred Terms) for analysis in the present study. PTs are the specific term used for a specific symptom, disease, therapeutic indication, medical or surgical procedures, family history characteristics, etc. PTs are grouped into SOC as per the etiology (eg, infections and infestations), manifestation sites (eg, gastrointestinal disorders, cardiac disorders, vascular disorders), or purpose (eg, surgical and medical procedures). ${ }^{26}$ The seriousness of the adverse event was decided as per the ICH E2B criteria, which identifies SAEs as those leading to either life-threatening event, hospitalization, disability, congenital abnormality, or death. ${ }^{27}$

This study included all the adverse events notified concerning all the suspected cardiovascular adverse events reported in VigiBase after administering any of the three COVID-19 vaccines: BNT162b2 Pfizer, 1222 AstraZeneca, and Moderna between December 15, 2020, and January 24, 2021. Since these three vaccines were being used thus only, the vaccines mentioned earlier were included for analysis in the present study. In the present study, we extracted three SOCs - cardiac disorders, vascular disorders, and investigations. The SOCInvestigation was further cleaned to remove all PTs other than those related to the cardiac and vascular systems. The individual cardiovascular adverse event is reported as serious and non-serious events in the form of frequency and percentage (Table 1). ECG changes are reported as frequency and percentage (Table 2). We also used disproportionality analysis, a method of signal detection for adverse events reported spontaneously in the database (Table 3). In this method, Frequentist [Reporting Odds Ratio (ROR), Proportional Reporting Ratio (PRR)] and Bayesian (Information Component (IC)) methods are used to compare drug adverse event pair with other drug adverse event pairs from the database to see if the observed frequency of the events for a drug is more than expected. ${ }^{28-32}$ We used Information Component (IC) value to evaluate the association between specific adverse events to COVID-19 vaccine administration. IC is the Bayesian method of signal generation and can avoid false-positive results even if the events are low. ${ }^{28}$ To link a particular adverse event to a drug (COVID-19 vaccine in this study), the lower limit of IC, ie, $\mathrm{IC}_{025}$, should have positive values. In this study, many events had a frequency of less than four; hence we used only $\mathrm{IC}_{025}$ values for this study, not the ROR or PRR. Though we did not use ROR and (PRR to link the events with vaccines for the event with $\mathrm{IC}_{025}$ positive, we have also mentioned the ROR and PRR values with 95\% Credibility Interval. The $\mathrm{IC}_{025}$ value was calculated separately for both the genders and different age groups.

\section{Statistical Analysis}

Descriptive statistics were reported in the form of frequency and percentage. Statistical Package for Social Science version 17 was used for analysis.

\section{Ethical Approval}

Institutional Ethics Committee exempted this project from the ethics review as this study is based on secondary data analysis, which involves no direct contact with any human subject.

\section{Results}

There were 103,954 adverse events reported for 30,523 patients from 15th December 2020 to 24th January 2021 (Figure 1). Most of the adverse events (AEs) were reported for three vaccines: BNT162b2 Pfizer, 1222 AstraZeneca, and Moderna COVID-19 vaccines since during the study period, these vaccines were used more commonly. Only one AE was reported from the AG0301-COVID19 vaccine and 32 from SARS-CoV-2 Vaccine (Vero Cell) Inactivated vaccine; none of these AEs were related to the cardiovascular system. A total of 4863 cardiovascular adverse events were reported from the COVID-19 vaccines. Adverse events as clinical endpoints and electrocardiographic endpoints reported from COVID-19 vaccines are mentioned in Tables 1 and 2. The adverse events related to a specific vaccine are given as Supplementary Tables 1-6. Common adverse events observed with vaccines under study were tachycardia (16.41\%), flushing (12.17\%). Hypertension $(5.82 \%)$, hypotension $(3.60 \%)$ and peripheral coldness $(2.41 \%)$. There were nine cardiovascular clinical endpoints and four electrocardiographic endpoints reported from the vaccines whose descriptions were not mentioned in the database. Amongst the clinical endpoints reported from unknown vaccines, there were events of four tachycardia (one serious), one decrease in blood pressure (serious), one increase in the blood pressure, one flushing (serious), and one hypertension. There were reports of four tachycardia from the electrocardiographic endpoints, one of which was serious in nature.

According to the disproportionality analysis based on $\mathrm{IC}_{025}$ values, acute myocardial infarction, cardiac arrest, and circulatory collapse were associated with the vaccine 
Table I Cardiovascular Related Adverse Drug Events Suspected to Be Caused by COVID-I9 Vaccines Use

\begin{tabular}{|c|c|c|c|c|}
\hline Broad Heading & Specific Adverse Event & $\begin{array}{l}\text { Non- Serious } n(\%) \\
(n=3264)\end{array}$ & $\begin{array}{l}\text { Serious } n(\%) \\
(n=1599)\end{array}$ & $\begin{array}{l}\text { Total n (\%) } \\
(n=4863)\end{array}$ \\
\hline \multirow{33}{*}{$\begin{array}{l}\text { Cardiac Disorder } \\
(\mathrm{N}=1995)\end{array}$} & Acute coronary syndrome & $0(0.00)$ & $5(0.3 I)$ & $5(0.10)$ \\
\hline & Acute myocardial infarction & $I(0.03)$ & $15(0.94)$ & $16(0.33)$ \\
\hline & Angina pectoris & $8(0.25)$ & $5(0.31)$ & $13(0.27)$ \\
\hline & Aortic valve calcification & $0(0.00)$ & $I(0.06)$ & $I(0.02)$ \\
\hline & Arrhythmia & $14(0.43)$ & $6(0.38)$ & $20(0.4 I)$ \\
\hline & Arrhythmia supraventricular & $2(0.06)$ & $0(0.00)$ & $2(0.04)$ \\
\hline & Arteriosclerosis & $0(0.00)$ & $3(0.19)$ & $3(0.06)$ \\
\hline & Arteriospasm coronary & $0(0.00)$ & $I(0.06)$ & $I(0.02)$ \\
\hline & Atrial enlargement & $0(0.00)$ & $I(0.06)$ & $I(0.02)$ \\
\hline & Atrial fibrillation & $9(0.28)$ & $26(1.63)$ & $35(0.72)$ \\
\hline & Atrial flutter & $I(0.03)$ & $I(0.06)$ & $2(0.04)$ \\
\hline & Atrial tachycardia & $0(0.00)$ & $2(0.13)$ & $2(0.04)$ \\
\hline & Atrioventricular block complete & $0(0.00)$ & $2(0.13)$ & $2(0.04)$ \\
\hline & Blood pressure abnormal & $0(0.00)$ & $\mathrm{I}(0.06)$ & $\mathrm{I}(0.02)$ \\
\hline & Bradycardia & $13(0.40)$ & $27(1.69)$ & $40(0.82)$ \\
\hline & Bundle branch block right & $0(0.00)$ & $I(0.06)$ & $I(0.02)$ \\
\hline & Cardiac arrest & $I(0.03)$ & $34(2.13)$ & $35(0.72)$ \\
\hline & Cardiac discomfort & $4(0.12)$ & $2(0.13)$ & $6(0.12)$ \\
\hline & Cardiac disorder & $I(0.03)$ & $7(0.44)$ & $8(0.16)$ \\
\hline & Cardiac failure & $0(0.00)$ & $6(0.38)$ & $6(0.12)$ \\
\hline & Cardiac failure acute & $0(0.00)$ & $I(0.06)$ & $I(0.02)$ \\
\hline & Cardiac failure congestive & $0(0.00)$ & $2(0.13)$ & $2(0.04)$ \\
\hline & Cardiac fibrillation & $0(0.00)$ & $I(0.06)$ & $I(0.02)$ \\
\hline & Cardiac flutter & $13(0.40)$ & $2(0.13)$ & $15(0.31)$ \\
\hline & Cardiac valve disease & $0(0.00)$ & $I(0.06)$ & $I(0.02)$ \\
\hline & Cardiogenic shock & $0(0.00)$ & $I(0.06)$ & $I(0.02)$ \\
\hline & Cardiomegaly & $0(0.00)$ & $2(0.13)$ & $2(0.04)$ \\
\hline & Cardiopulmonary failure & $0(0.00)$ & $2(0.13)$ & $2(0.04)$ \\
\hline & Cardio-respiratory arrest & $0(0.00)$ & $4(0.25)$ & $4(0.08)$ \\
\hline & Cardio-respiratory distress & $0(0.00)$ & $\mathrm{I}(0.06)$ & $\mathrm{I}(0.02)$ \\
\hline & Cardiovascular disorder & $5(0.15)$ & $7(0.44)$ & $12(0.25)$ \\
\hline & Catheterisation cardiac abnormal & $0(0.00)$ & $I(0.06)$ & $\mathrm{I}(0.02)$ \\
\hline & Coronary artery disease & $0(0.00)$ & $2(0.13)$ & $2(0.04)$ \\
\hline
\end{tabular}

(Continued) 
Table I (Continued).

\begin{tabular}{|c|c|c|c|c|}
\hline Broad Heading & Specific Adverse Event & $\begin{array}{l}\text { Non- Serious } n(\%) \\
(n=3264)\end{array}$ & $\begin{array}{l}\text { Serious } n(\%) \\
(n=1599)\end{array}$ & $\begin{array}{l}\text { Total n (\%) } \\
(n=4863)\end{array}$ \\
\hline & Coronary artery occlusion & $0(0.00)$ & $2(0.13)$ & $2(0.04)$ \\
\hline & Coronary artery stenosis & $0(0.00)$ & $\mathrm{I}(0.06)$ & $\mathrm{I}(0.02)$ \\
\hline & C-reactive protein increased & $0(0.00)$ & $8(0.50)$ & $8(0.16)$ \\
\hline & Echocardiogram abnormal & $0(0.00)$ & $\mathrm{I}(0.06)$ & $\mathrm{I}(0.02)$ \\
\hline & Ejection fraction decreased & $0(0.00)$ & $2(0.13)$ & $2(0.04)$ \\
\hline & Electrocardiogram PR shortened & $0(0.00)$ & $\mathrm{I}(0.06)$ & $\mathrm{I}(0.02)$ \\
\hline & Electrocardiogram $\mathrm{Q}$ wave abnormal & $0(0.00)$ & $\mathrm{I}(0.06)$ & $\mathrm{I}(0.02)$ \\
\hline & Electrocardiogram ST segment elevation & $0(0.00)$ & $\mathrm{I}(0.06)$ & $I(0.02)$ \\
\hline & Electrocardiogram T wave abnormal & $0(0.00)$ & $\mathrm{I}(0.06)$ & $\mathrm{I}(0.02)$ \\
\hline & Extrasystoles & $12(0.37)$ & $7(0.44)$ & $19(0.39)$ \\
\hline & Fibrin $\mathrm{D}$ dimer increased & $0(0.00)$ & $2(0.13)$ & $2(0.04)$ \\
\hline & Heart sounds abnormal & $0(0.00)$ & $\mathrm{I}(0.06)$ & $\mathrm{I}(0.02)$ \\
\hline & International normalised ratio increased & $0(0.00)$ & $2(0.13)$ & $2(0.04)$ \\
\hline & $\begin{array}{l}\text { Left ventricular end-diastolic pressure } \\
\text { increased }\end{array}$ & $0(0.00)$ & $\mathrm{I}(0.06)$ & $\mathrm{I}(0.02)$ \\
\hline & Myocardial infarction & $2(0.06)$ & $30(1.88)$ & $32(0.66)$ \\
\hline & Myocardial ischaemia & $0(0.00)$ & $\mathrm{I}(0.06)$ & $\mathrm{I}(0.02)$ \\
\hline & Myocarditis & $\mathrm{I}(0.03)$ & $5(0.31)$ & $6(0.12)$ \\
\hline & Palpitations & $532(16.30)$ & $185(11.57)$ & $717(14.74)$ \\
\hline & Pericarditis & $2(0.06)$ & $5(0.31)$ & $7(0.14)$ \\
\hline & $\begin{array}{l}\text { Postural orthostatic tachycardia } \\
\text { syndrome }\end{array}$ & $0(0.00)$ & $2(0.13)$ & $2(0.04)$ \\
\hline & Prothrombin time prolonged & $0(0.00)$ & $\mathrm{I}(0.06)$ & $\mathrm{I}(0.02)$ \\
\hline & Right ventricular enlargement & $0(0.00)$ & $\mathrm{I}(0.06)$ & $\mathrm{I}(0.02)$ \\
\hline & Sinus bradycardia & $I(0.03)$ & $2(0.13)$ & $3(0.06)$ \\
\hline & Sinus tachycardia & $36(1.10)$ & $23(1.44)$ & $59(1.21)$ \\
\hline & Stress cardiomyopathy & $0(0.00)$ & $2(0.13)$ & $2(0.04)$ \\
\hline & Supraventricular extrasystoles & $6(0.18)$ & $0(0.00)$ & $6(0.12)$ \\
\hline & Supraventricular tachycardia & $10(0.31)$ & 19(1.19) & $29(0.60)$ \\
\hline & Tachyarrhythmia & $I(0.03)$ & $5(0.31)$ & $6(0.12)$ \\
\hline & Tachycardia & $557(17.06)$ & $24 I(15.07)$ & $798(16.4 I)$ \\
\hline & Tachycardia paroxysmal & $3(0.09)$ & $0(0.00)$ & $3(0.06)$ \\
\hline & Tricuspid valve incompetence & $0(0.00)$ & $\mathrm{I}(0.06)$ & $\mathrm{I}(0.02)$ \\
\hline & Troponin increased & $0(0.00)$ & $5(0.3 I)$ & $5(0.10)$ \\
\hline
\end{tabular}

(Continued) 
Table I (Continued).

\begin{tabular}{|c|c|c|c|c|}
\hline Broad Heading & Specific Adverse Event & $\begin{array}{l}\text { Non- Serious n (\%) } \\
(n=3264)\end{array}$ & $\begin{array}{l}\text { Serious } n(\%) \\
(n=1599)\end{array}$ & $\begin{array}{l}\text { Total n (\%) } \\
(n=4863)\end{array}$ \\
\hline & Ventricular arrhythmia & $0(0.00)$ & $2(0.13)$ & $2(0.04)$ \\
\hline & Ventricular extrasystoles & $15(0.46)$ & $4(0.25)$ & 19(0.39) \\
\hline & Ventricular fibrillation & $0(0.00)$ & $\mathrm{I}(0.06)$ & $\mathrm{I}(0.02)$ \\
\hline & Ventricular hypokinesia & $0(0.00)$ & $\mathrm{I}(0.06)$ & $\mathrm{I}(0.02)$ \\
\hline & Ventricular tachycardia & $0(0.00)$ & $5(0.31)$ & $5(0.10)$ \\
\hline \multirow[t]{27}{*}{ Investigations $(\mathrm{N}=1055)$} & $\begin{array}{l}\text { Activated partial thromboplastin time } \\
\text { prolonged }\end{array}$ & $0(0.00)$ & $\mathrm{I}(0.06)$ & $\mathrm{I}(0.02)$ \\
\hline & $\begin{array}{l}\text { Activated partial thromboplastin time } \\
\text { shortened }\end{array}$ & $\mathrm{I}(0.03)$ & $2(0.13)$ & $3(0.06)$ \\
\hline & Angiogram abnormal & $0(0.00)$ & $2(0.13)$ & $2(0.04)$ \\
\hline & Blood pressure abnormal & $9(0.28)$ & $3(0.19)$ & $12(0.25)$ \\
\hline & Blood pressure decreased & $24(0.74)$ & $20(1.25)$ & $44(0.90)$ \\
\hline & Blood pressure diastolic increased & $2(0.06)$ & $3(0.19)$ & $5(0.10)$ \\
\hline & Blood pressure immeasurable & $2(0.06)$ & $I(0.06)$ & $3(0.06)$ \\
\hline & Blood pressure increased & $255(7.81)$ & $62(3.88)$ & $317(6.52)$ \\
\hline & Blood pressure orthostatic & $\mathrm{I}(0.03)$ & $0(0.00)$ & $\mathrm{I}(0.02)$ \\
\hline & Blood pressure orthostatic abnormal & $I(0.03)$ & $0(0.00)$ & $\mathrm{I}(0.02)$ \\
\hline & Blood pressure orthostatic increased & $\mathrm{I}(0.03)$ & $0(0.00)$ & $\mathrm{I}(0.02)$ \\
\hline & Blood pressure systolic abnormal & $2(0.06)$ & $0(0.00)$ & $2(0.04)$ \\
\hline & Blood pressure systolic decreased & $3(0.09)$ & $0(0.00)$ & $3(0.06)$ \\
\hline & Blood pressure systolic increased & $3(0.09)$ & $4(0.25)$ & $7(0.14)$ \\
\hline & Cardiac monitoring abnormal & $I(0.03)$ & $3(0.19)$ & $4(0.08)$ \\
\hline & Cardiac ventriculogram left & $0(0.00)$ & $I(0.06)$ & $I(0.02)$ \\
\hline & Carotid bruit & $0(0.00)$ & $I(0.06)$ & $I(0.02)$ \\
\hline & Catheterisation cardiac abnormal & $0(0.00)$ & $2(0.13)$ & $2(0.04)$ \\
\hline & Central venous pressure & $I(0.03)$ & $0(0.00)$ & $I(0.02)$ \\
\hline & C-reactive protein decreased & $I(0.03)$ & $0(0.00)$ & $I(0.02)$ \\
\hline & C-reactive protein increased & $4(0.12)$ & II (0.69) & $15(0.31)$ \\
\hline & Echocardiogram abnormal & $0(0.00)$ & $I(0.06)$ & $I(0.02)$ \\
\hline & Ejection fraction decreased & $0(0.00)$ & $2(0.13)$ & $2(0.04)$ \\
\hline & Electrocardiogram abnormal & $21(0.64)$ & $13(0.8 \mid)$ & $34(0.70)$ \\
\hline & Electrocardiogram change & $I(0.03)$ & $0(0.00)$ & $I(0.02)$ \\
\hline & Electrocardiogram PR prolongation & $0(0.00)$ & $\mathrm{I}(0.06)$ & $\mathrm{I}(0.02)$ \\
\hline & Electrocardiogram QT prolonged & $3(0.09)$ & $0(0.00)$ & $3(0.06)$ \\
\hline
\end{tabular}

(Continued) 
Table I (Continued).

\begin{tabular}{|c|c|c|c|c|}
\hline Broad Heading & Specific Adverse Event & $\begin{array}{l}\text { Non- Serious } n(\%) \\
(n=3264)\end{array}$ & $\begin{array}{l}\text { Serious } n(\%) \\
(n=1599)\end{array}$ & $\begin{array}{l}\text { Total } n(\%) \\
(n=4863)\end{array}$ \\
\hline & $\begin{array}{l}\text { Electrocardiogram ST segment } \\
\text { depression }\end{array}$ & $0(0.00)$ & $\mathrm{I}(0.06)$ & $I(0.02)$ \\
\hline & Electrocardiogram ST segment elevation & $0(0.00)$ & $5(0.3 I)$ & $5(0.10)$ \\
\hline & Electrocardiogram T wave abnormal & $0(0.00)$ & $2(0.13)$ & $2(0.04)$ \\
\hline & Electrocardiogram $T$ wave inversion & $I(0.03)$ & $I(0.06)$ & $2(0.04)$ \\
\hline & Electrocardiogram T wave peaked & $0(0.00)$ & $\mathrm{I}(0.06)$ & $\mathrm{I}(0.02)$ \\
\hline & Electroencephalogram abnormal & $0(0.00)$ & $\mathrm{I}(0.06)$ & $\mathrm{I}(0.02)$ \\
\hline & Fibrin $D$ dimer increased & $4(0.12)$ & $10(0.63)$ & $14(0.29)$ \\
\hline & Foetal heart rate abnormal & $0(0.00)$ & $\mathrm{I}(0.06)$ & $\mathrm{I}(0.02)$ \\
\hline & Heart rate abnormal & $6(0.18)$ & $0(0.00)$ & $6(0.12)$ \\
\hline & Heart rate decreased & $15(0.46)$ & $8(0.50)$ & $23(0.47)$ \\
\hline & Heart rate increased & $336(10.29)$ & $103(6.44)$ & $439(9.03)$ \\
\hline & Heart rate irregular & $18(0.55)$ & $6(0.38)$ & $24(0.49)$ \\
\hline & Heart sounds abnormal & $I(0.03)$ & $0(0.00)$ & $\mathrm{I}(0.02)$ \\
\hline & International normalised ratio decreased & $\mathrm{I}(0.03)$ & $\mathrm{I}(0.06)$ & $2(0.04)$ \\
\hline & International normalised ratio increased & $2(0.06)$ & $8(0.50)$ & $10(0.21)$ \\
\hline & Myocardial necrosis marker increased & $0(0.00)$ & $2(0.13)$ & $2(0.04)$ \\
\hline & Prothrombin time prolonged & $0(0.00)$ & $2(0.13)$ & $2(0.04)$ \\
\hline & Prothrombin time shortened & $0(0.00)$ & $I(0.06)$ & $\mathrm{I}(0.02)$ \\
\hline & Pulse abnormal & 14(0.43) & $5(0.3 I)$ & $19(0.39)$ \\
\hline & Pulse absent & $0(0.00)$ & $16(1.00)$ & $16(0.33)$ \\
\hline & Troponin I increased & $\mathrm{I}(0.03)$ & $2(0.13)$ & $3(0.06)$ \\
\hline & Troponin increased & $0(0.00)$ & $9(0.56)$ & $9(0.19)$ \\
\hline & Troponin $\mathrm{T}$ increased & $0(0.00)$ & $2(0.13)$ & $2(0.04)$ \\
\hline \multirow{9}{*}{$\begin{array}{l}\text { Vascular Disorder } \\
(\mathrm{N}=1813)\end{array}$} & Aneurysm & $0(0.00)$ & $I(0.06)$ & $\mathrm{I}(0.02)$ \\
\hline & Angiopathy & $\mathrm{I}(0.03)$ & $0(0.00)$ & $\mathrm{I}(0.02)$ \\
\hline & Blood pressure fluctuation & $6(0.18)$ & $\mathrm{I}(0.06)$ & $7(0.14)$ \\
\hline & Capillary fragility & $0(0.00)$ & $2(0.13)$ & $2(0.04)$ \\
\hline & Circulatory collapse & $5(0.15)$ & $21(1.31)$ & $26(0.53)$ \\
\hline & Deep vein thrombosis & $3(0.09)$ & $7(0.44)$ & $10(0.21)$ \\
\hline & Diastolic hypertension & $0(0.00)$ & $I(0.06)$ & $I(0.02)$ \\
\hline & Flushing & $487(\mid 4.92)$ & $105(6.57)$ & $592(12.17)$ \\
\hline & Haematoma & $I(0.03)$ & $0(0.00)$ & $I(0.02)$ \\
\hline
\end{tabular}

(Continued) 
Table I (Continued).

\begin{tabular}{|c|c|c|c|c|}
\hline Broad Heading & Specific Adverse Event & $\begin{array}{l}\text { Non- Serious } n(\%) \\
(n=3264)\end{array}$ & $\begin{array}{l}\text { Serious } n(\%) \\
(n=1599)\end{array}$ & $\begin{array}{l}\text { Total } n(\%) \\
(n=4863)\end{array}$ \\
\hline & Haemorrhage & II (0.34) & $7(0.44)$ & $18(0.37)$ \\
\hline & Hot flush & $227(6.95)$ & $77(4.82)$ & $304(6.25)$ \\
\hline & Hypertension & $190(5.82)$ & $93(5.82)$ & $283(5.82)$ \\
\hline & Hypertensive crisis & $13(0.40)$ & $15(0.94)$ & $28(0.58)$ \\
\hline & Hypertensive emergency & $4(0.12)$ & $\mathrm{I}(0.06)$ & $5(0.10)$ \\
\hline & Hypertensive urgency & $\mathrm{I}(0.03)$ & $2(0.13)$ & $3(0.06)$ \\
\hline & Hypoperfusion & $0(0.00)$ & $\mathrm{I}(0.06)$ & $I(0.02)$ \\
\hline & Hypotension & $86(2.63)$ & $89(5.57)$ & $175(3.60)$ \\
\hline & Hypotensive crisis & $2(0.06)$ & $0(0.00)$ & $2(0.04)$ \\
\hline & Hypovolaemic shock & $0(0.00)$ & $\mathrm{I}(0.06)$ & $I(0.02)$ \\
\hline & Labile blood pressure & $I(0.03)$ & $0(0.00)$ & $I(0.02)$ \\
\hline & Microembolism & $\mathrm{I}(0.03)$ & $0(0.00)$ & $\mathrm{I}(0.02)$ \\
\hline & Neurogenic shock & $4(0.12)$ & $\mathrm{I}(0.06)$ & $5(0.10)$ \\
\hline & Orthostatic hypotension & $7(0.21)$ & $3(0.19)$ & $10(0.21)$ \\
\hline & Pallor & $101(3.09)$ & $38(2.38)$ & $139(2.86)$ \\
\hline & Pelvic venous thrombosis & $0(0.00)$ & $\mathrm{I}(0.06)$ & $\mathrm{I}(0.02)$ \\
\hline & Peripheral circulatory failure & $4(0.12)$ & $2(0.13)$ & $6(0.12)$ \\
\hline & Peripheral coldness & $86(2.63)$ & $31(1.94)$ & $117(2.41)$ \\
\hline & Peripheral ischaemia & $0(0.00)$ & $2(0.13)$ & $2(0.04)$ \\
\hline & Peripheral vascular disorder & $13(0.40)$ & $5(0.31)$ & $18(0.37)$ \\
\hline & Phlebitis & $9(0.28)$ & $2(0.13)$ & $\mathrm{II}(0.23)$ \\
\hline & Poor peripheral circulation & $2(0.06)$ & $\mathrm{I}(0.06)$ & $3(0.06)$ \\
\hline & Raynaud's phenomenon & $3(0.09)$ & $5(0.31)$ & $8(0.16)$ \\
\hline & Shock & $0(0.00)$ & $\mathrm{I}(0.06)$ & $\mathrm{I}(0.02)$ \\
\hline & Systolic hypertension & $0(0.00)$ & $\mathrm{I}(0.06)$ & $I(0.02)$ \\
\hline & Thrombophlebitis & $I(0.03)$ & $2(0.13)$ & $3(0.06)$ \\
\hline & Thrombosis & $I(0.03)$ & $6(0.38)$ & $7(0.14)$ \\
\hline & Varicose vein & $\mathrm{I}(0.03)$ & $0(0.00)$ & $\mathrm{I}(0.02)$ \\
\hline & Vascular occlusion & $I(0.03)$ & $0(0.00)$ & $I(0.02)$ \\
\hline & Vasculitis & $\mathrm{I}(0.03)$ & $2(0.13)$ & $3(0.06)$ \\
\hline & Vasoconstriction & $\mathrm{I}(0.03)$ & $2(0.13)$ & $3(0.06)$ \\
\hline & Vasodilatation & $\mathrm{I}(0.03)$ & $\mathrm{I}(0.06)$ & $2(0.04)$ \\
\hline & Vein discolouration & $2(0.06)$ & $0(0.00)$ & $2(0.04)$ \\
\hline
\end{tabular}

(Continued) 
Table I (Continued).

\begin{tabular}{|c|c|c|c|c|}
\hline Broad Heading & Specific Adverse Event & $\begin{array}{l}\text { Non- Serious } n(\%) \\
(n=3264)\end{array}$ & $\begin{array}{l}\text { Serious } n(\%) \\
(n=1599)\end{array}$ & $\begin{array}{l}\text { Total n (\%) } \\
(n=4863)\end{array}$ \\
\hline & Vein disorder & $0(0.00)$ & $2(0.13)$ & $2(0.04)$ \\
\hline & Vein rupture & $0(0.00)$ & $\mathrm{I}(0.06)$ & $\mathrm{I}(0.02)$ \\
\hline & Venous thrombosis limb & $0(0.00)$ & $\mathrm{I}(0.06)$ & $\mathrm{I}(0.02)$ \\
\hline & White coat hypertension & $\mathrm{I}(0.03)$ & $0(0.00)$ & $\mathrm{I}(0.02)$ \\
\hline & Withdrawal hypertension & $\mathrm{I}(0.03)$ & $0(0.00)$ & $\mathrm{I}(0.02)$ \\
\hline
\end{tabular}

used in the age group $>75$ years. Hypertension and severe hypertension like hypertensive emergency and urgency were associated with vaccine use in almost all age groups and genders. Vaccines were also associated with rhythm disorders such as supraventricular tachycardia, sinus tachycardia, paroxysmal tachycardia, palpitations, etc. Peripheral circular failure was also found to be associated with vaccine use. Amongst the investigations and lab tests, abnormal ECG findings, raised C-reactive protein, increase in D-dimer, increase in troponin was found to be associated with the vaccine used in specific age group or gender or all subjects (Table 3 ).

\section{Discussion}

We explored the global pharmacovigilance database VigiBase for cardiovascular adverse events reported for COVID-19 vaccines in the present study. The majority of the present study's adverse events were BNT162b2 Pfizer, 1222 AstraZeneca, and Moderna vaccines. The elderly age group was associated with acute myocardial infarction, cardiac arrest, and circulatory collapse. Hypertension, severe hypertension, supraventricular tachycardia, sinus tachycardia, paroxysmal tachycardia, palpitations, etc., were observed in all age groups and both genders. Abnormal ECG findings, elevated C-reactive protein, elevated D-dimer, and troponin associated with the vaccine used in a specific age group or gender or all subjects.

The current analysis shows the total number of cardiovascular adverse events was higher in individuals receiving Pfizer-BioNTech COVID-19 (BNT162b2) vaccine, although the values might not reflect the real situation as it was the first vaccine to get emergency approval from USFDA; hence a large number of doses have been administered till the period of analysis, and other reason could be a shorter interval between the two doses. ${ }^{33}$
According to the disproportionality analysis based on $\mathrm{IC}_{025}$ values, acute myocardial infarction, cardiac arrest, and circulatory collapse were associated with the vaccine used in the age group $>75$ years. The Case Series Drug Analysis Print published by AstraZeneca for their COVID19 vaccine as of May 20, 2021, reported a total of 6959 cardiac AEs, among which 4106 events were of palpitations, 1099 were of tachycardia, 129 events of cardiac arrest, 246 events of myocardial infarction, 220 events of atrial fibrillation, 154 events of cardiac flutter, 142 events of angina pectoris, 91 events of arrhythmia, 59 events of sinus tachycardia, 44 events of acute myocardial infarction and 45 events of cardiac failure. ${ }^{34}$ The data reported from the clinical trials by Moderna in the FDA Briefing Document shows myocardial infarction was reported in $0.03 \%$ of cases in the vaccine group. ${ }^{35}$ Similarly, the data reported by Pfizer-BioNTech COVID-19 Vaccine in the FDA Briefing Document showed one event of cardiac arrest, one event of ventricular arrhythmias, and events of acute myocardial infarction in $0.02 \%$ of the patients. ${ }^{36}$ The Case Series Drug Analysis Print published by PfizerBioNTech for their COVID-19 vaccine as of May 28, 2021, reported a total of 2342 cardiac AEs, among which 1098 events were of palpitation, 466 events of tachycardia, 108 events of atrial fibrillation, 94 events of myocardial infarction, 62 events of cardiac arrest, 63 events of cardiac flutter, 32 events of sinus tachycardia, 46 events of angina pectoris, 24 events of cardiac failure, 38 events of arrhythmia and 16 events of acute myocardial infarction. ${ }^{37}$

In the COVID-19 vaccine safety update by Advisory Committee on Immunization Practices (ACIP), the Centers for Disease Control and Prevention (CDC) and the FDA, as of Jan 27, 2021, reported a single event of acute myocardial infarction. ${ }^{38} \mathrm{~A}$ case report by Boivin et al reported myocardial infarction with a 96 years old female and no known cardiac history with the Moderna COVID-19 
Table 2 Analysis of Electrocardiographic Findings Among Reported Adverse Drug Events Associated with COVID-19 Vaccines

\begin{tabular}{|c|c|c|c|c|}
\hline $\begin{array}{l}\text { Electrocardiogram } \\
\text { Findings }\end{array}$ & Specific Findings & Non- Serious n (\%) (N=720) & Serious $n(\%)(N=4 \mid 0)$ & Total n (\%) (N= I I 30 \\
\hline \multirow[t]{4}{*}{ Bradyarrhythmias } & Arrhythmia & 14(1.94) & $6(1.46)$ & $20(1.77)$ \\
\hline & Arrhythmia supraventricular & $2(0.28)$ & $0(0.00)$ & $2(0.18)$ \\
\hline & Atrial fibrillation & $9(1.25)$ & $26(6.34)$ & $35(3.10)$ \\
\hline & Atrial flutter & $\mathrm{I}(0.14)$ & $\mathrm{I}(0.24)$ & $2(0.18)$ \\
\hline \multirow[t]{12}{*}{ Tachyarrhythmias } & Sinus bradycardia & $\mathrm{I}(0.14)$ & $2(0.49)$ & $3(0.27)$ \\
\hline & Sinus tachycardia & $36(5.00)$ & $23(5.61)$ & $59(5.22)$ \\
\hline & Supraventricular extrasystoles & $6(0.83)$ & $0(0.00)$ & $6(0.53)$ \\
\hline & Supraventricular tachycardia & $10(1.39)$ & $19(4.63)$ & $29(2.57)$ \\
\hline & Tachyarrhythmia & $\mathrm{I}(0.14)$ & $5(1.22)$ & $6(0.53)$ \\
\hline & Tachycardia & $557(77.36)$ & $24 I(58.78)$ & $798(70.62)$ \\
\hline & Tachycardia paroxysmal & $3(0.42)$ & $0(0.00)$ & $3(0.27)$ \\
\hline & Ventricular arrhythmia & $0(0.00)$ & $2(0.49)$ & $2(0.18)$ \\
\hline & Ventricular extrasystoles & $15(2.08)$ & $4(0.98)$ & $19(1.68)$ \\
\hline & Ventricular fibrillation & $0(0.00)$ & $\mathrm{I}(0.24)$ & $I(0.09)$ \\
\hline & Ventricular hypokinesia & $0(0.00)$ & $\mathrm{I}(0.24)$ & $I(0.09)$ \\
\hline & Ventricular tachycardia & $0(0.00)$ & $5(1.22)$ & $5(0.44)$ \\
\hline \multirow[t]{7}{*}{ Non-Specific } & Electrocardiogram QT prolonged & $3(0.42)$ & $0(0.00)$ & $3(0.27)$ \\
\hline & $\begin{array}{l}\text { Electrocardiogram ST segment } \\
\text { depression }\end{array}$ & $0(0.00)$ & $\mathrm{I}(0.24)$ & $I(0.09)$ \\
\hline & Electrocardiogram ST segment elevation & $0(0.00)$ & $6(1.46)$ & $6(0.53)$ \\
\hline & Electrocardiogram T wave abnormal & $0(0.00)$ & $3(0.73)$ & $3(0.27)$ \\
\hline & Electrocardiogram T wave inversion & $\mathrm{I}(0.14)$ & $\mathrm{I}(0.24)$ & $2(0.18)$ \\
\hline & Electrocardiogram T wave peaked & $0(0.00)$ & $\mathrm{I}(0.24)$ & $\mathrm{I}(0.09)$ \\
\hline & Extrasystoles & $12(1.67)$ & $7(1.71)$ & $19(1.68)$ \\
\hline \multirow[t]{12}{*}{ Miscellaneous } & Atrial tachycardia & $0(0.00)$ & $2(0.49)$ & $2(0.18)$ \\
\hline & Atrioventricular block complete & $0(0.00)$ & $2(0.49)$ & $2(0.18)$ \\
\hline & Bradycardia & $13(1.81)$ & $27(6.59)$ & $40(3.54)$ \\
\hline & Bundle branch block right & $0(0.00)$ & $\mathrm{I}(0.24)$ & I (0.09) \\
\hline & Cardiac fibrillation & $0(0.00)$ & $\mathrm{I}(0.24)$ & I (0.09) \\
\hline & Cardiac flutter & $13(1.81)$ & $2(0.49)$ & $15(1.33)$ \\
\hline & Cardiac monitoring abnormal & $\mathrm{I}(0.14)$ & $3(0.73)$ & $4(0.35)$ \\
\hline & Echocardiogram abnormal & $0(0.00)$ & $\mathrm{I}(0.24)$ & I (0.09) \\
\hline & Electrocardiogram abnormal & $22(3.06)$ & $13(3.17)$ & $35(3.10)$ \\
\hline & Electrocardiogram PR prolongation & $0(0.00)$ & $\mathrm{I}(0.24)$ & $\mathrm{I}(0.09)$ \\
\hline & Electrocardiogram PR shortened & $0(0.00)$ & $\mathrm{I}(0.24)$ & $I(0.09)$ \\
\hline & Electrocardiogram Q wave abnormal & $0(0.00)$ & $\mathrm{I}(0.24)$ & $\mathrm{I}(0.09)$ \\
\hline
\end{tabular}




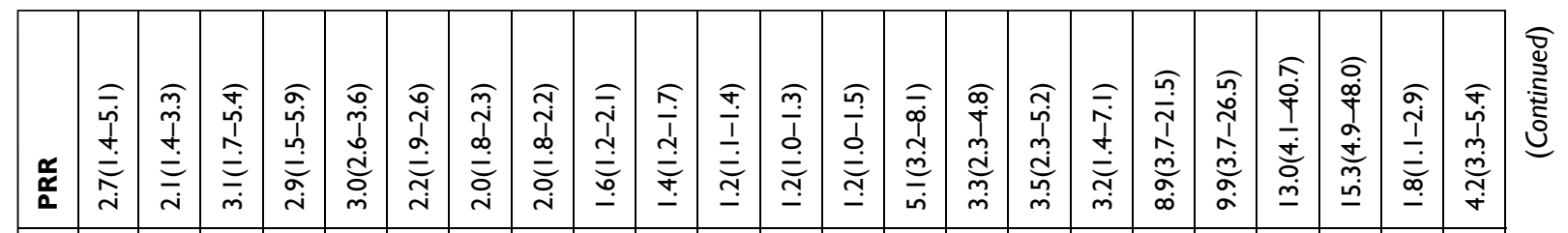

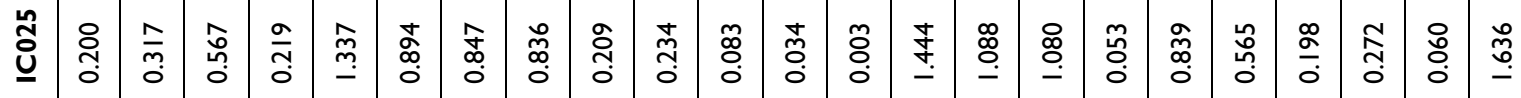

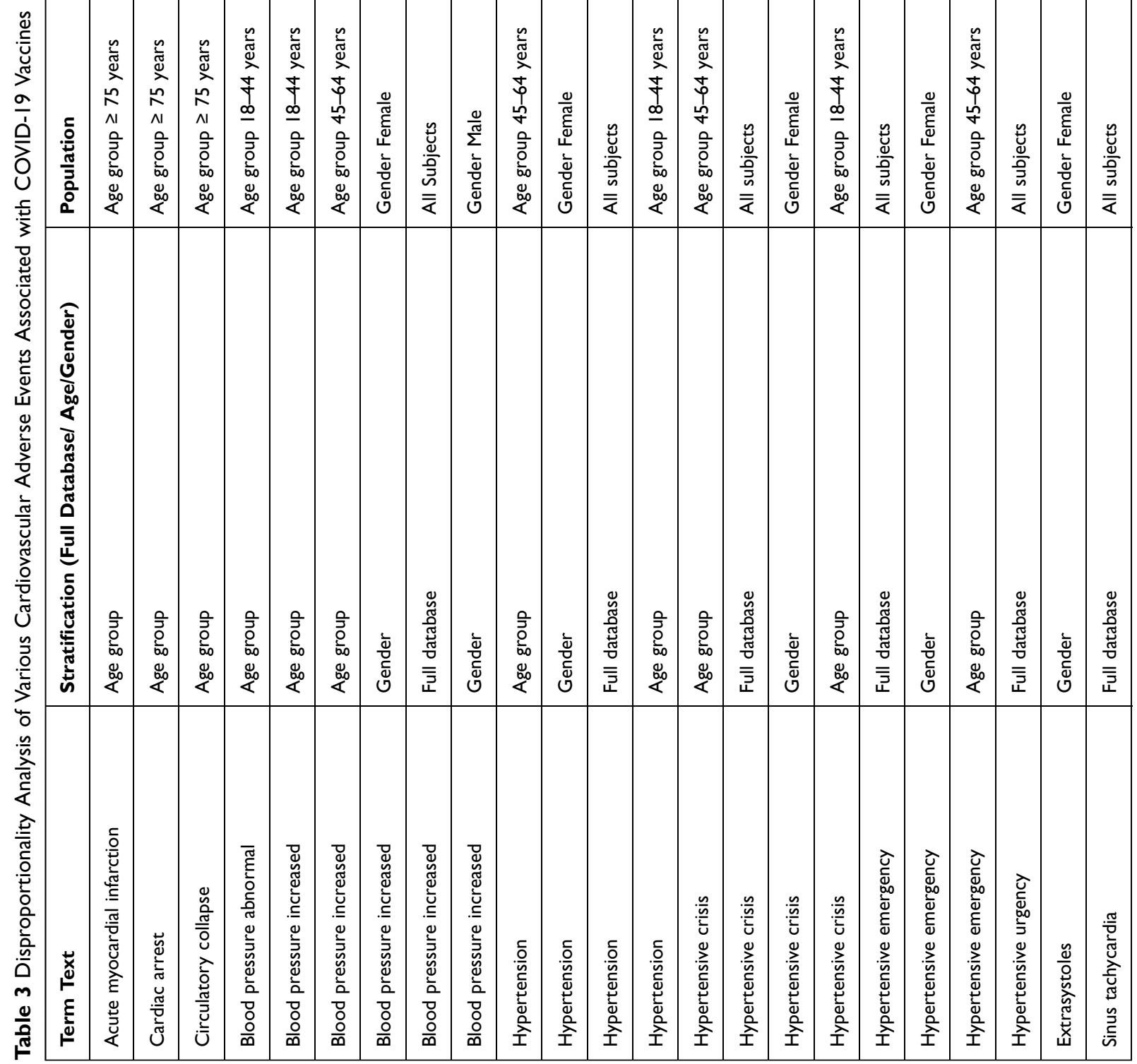




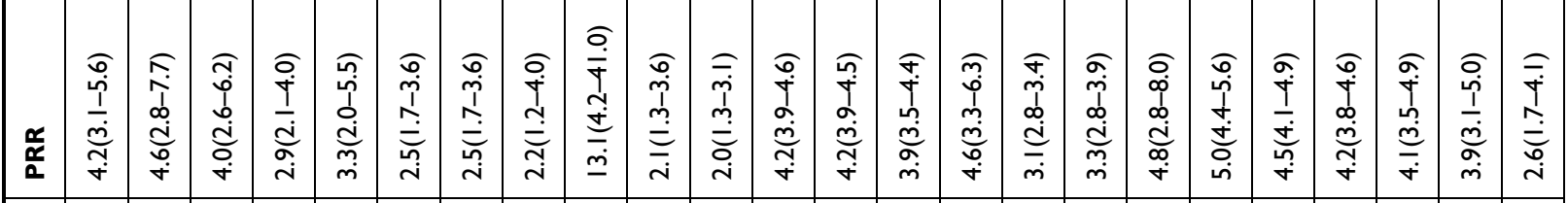

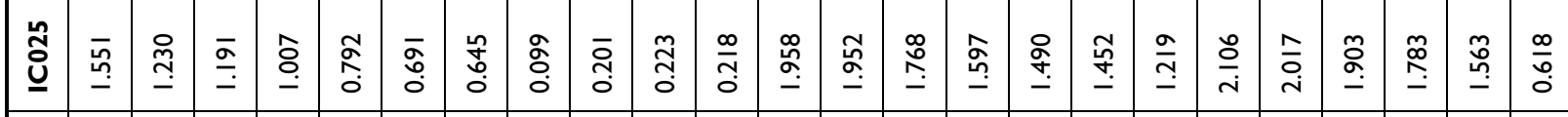

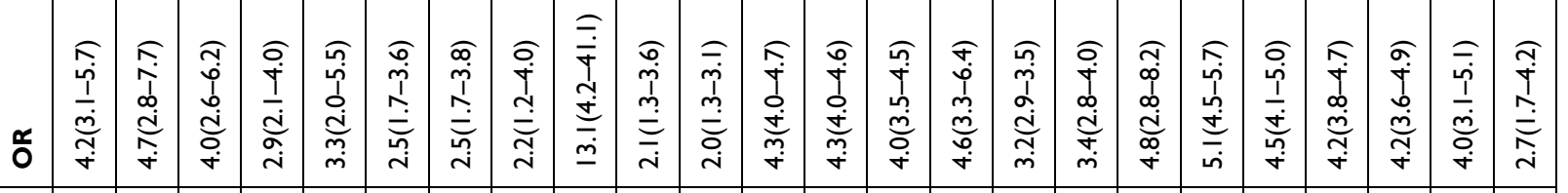

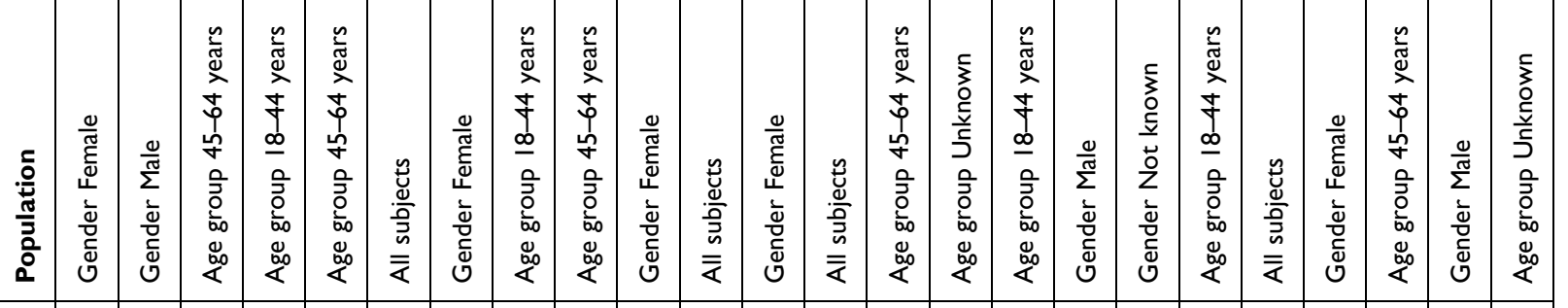

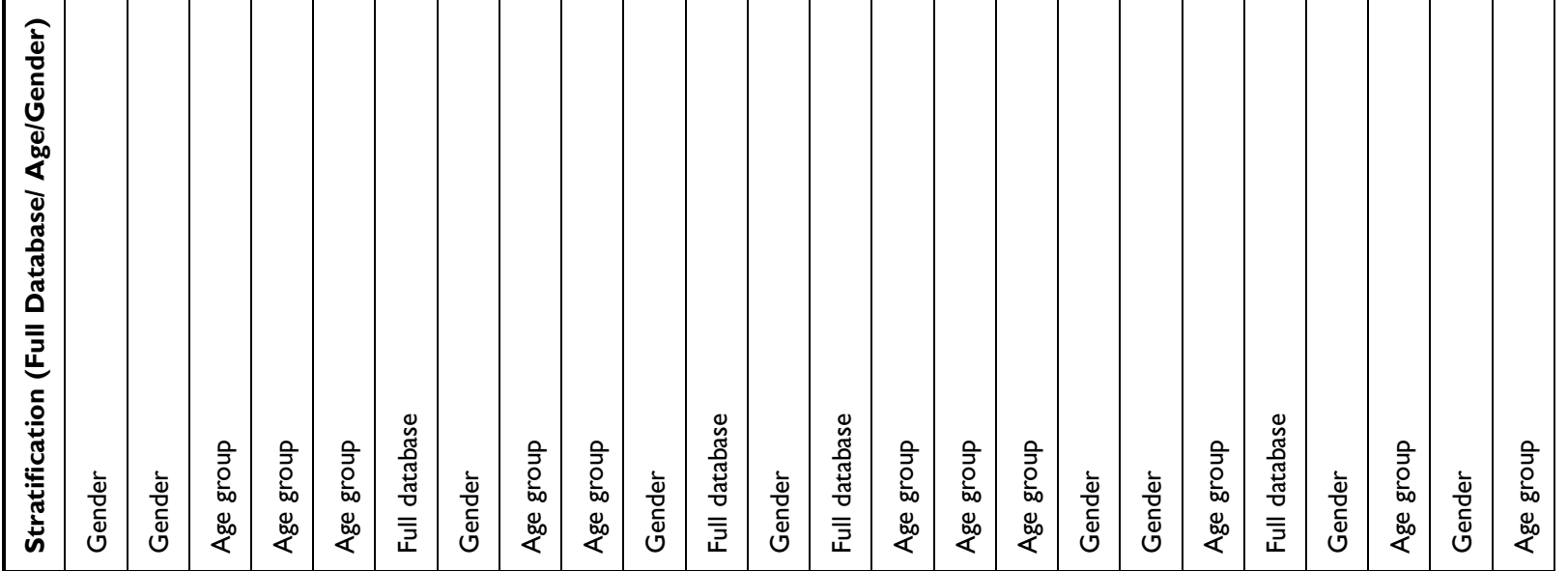

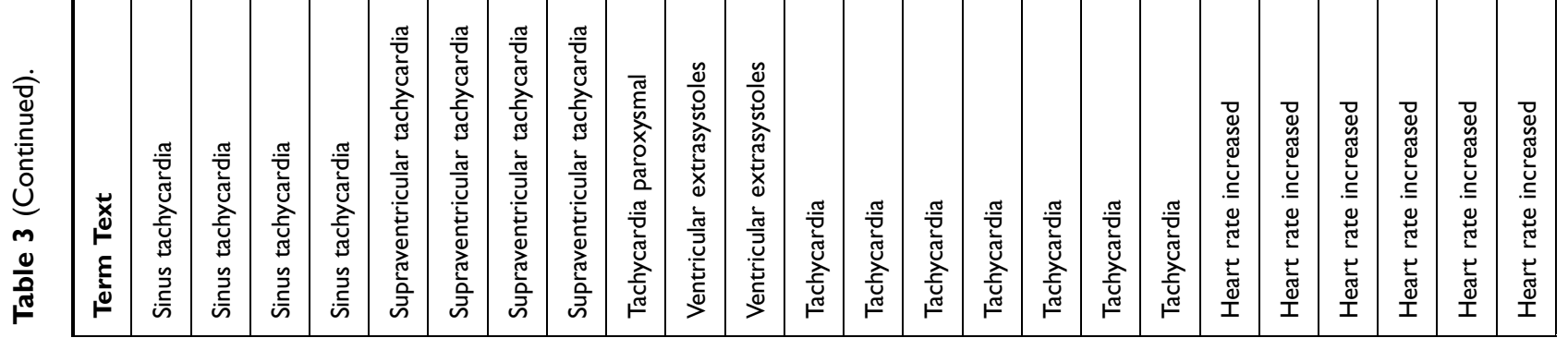




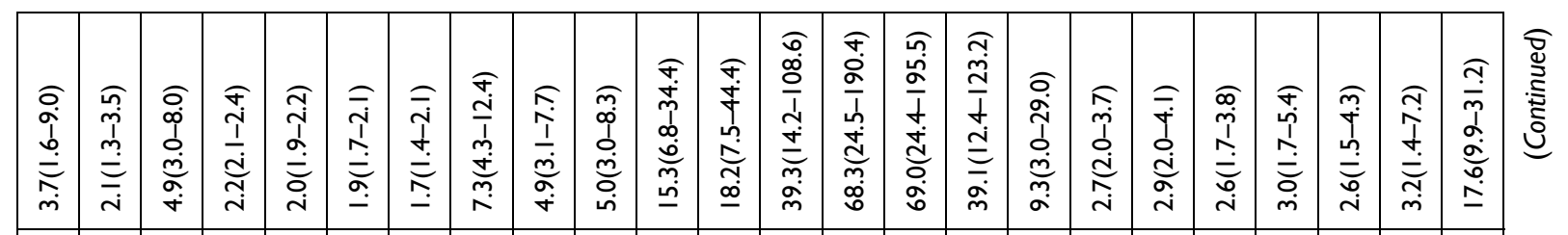

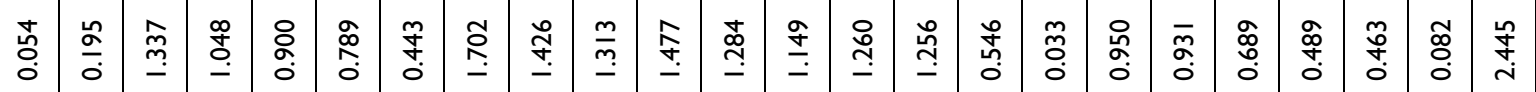

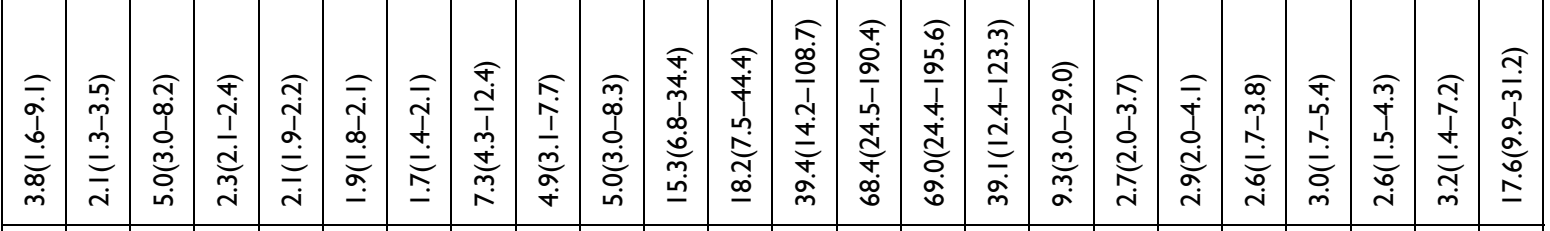

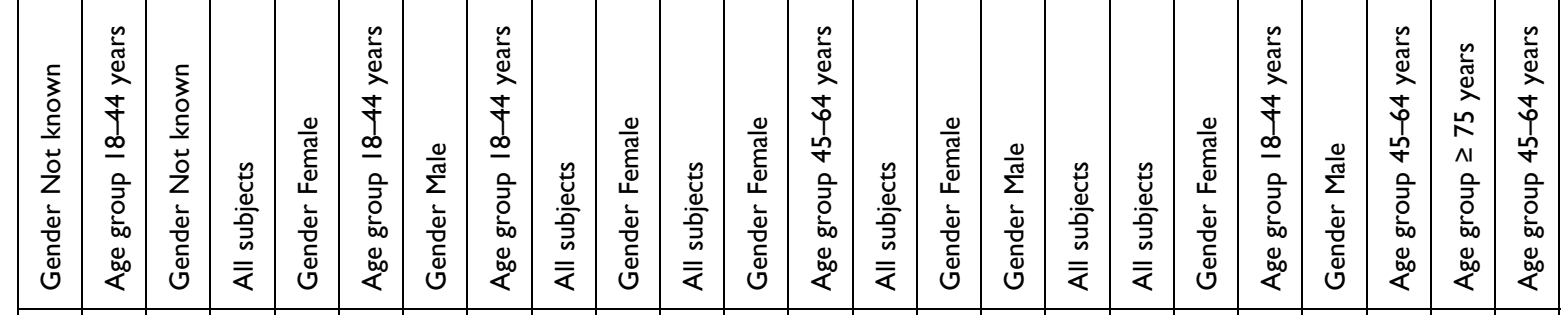

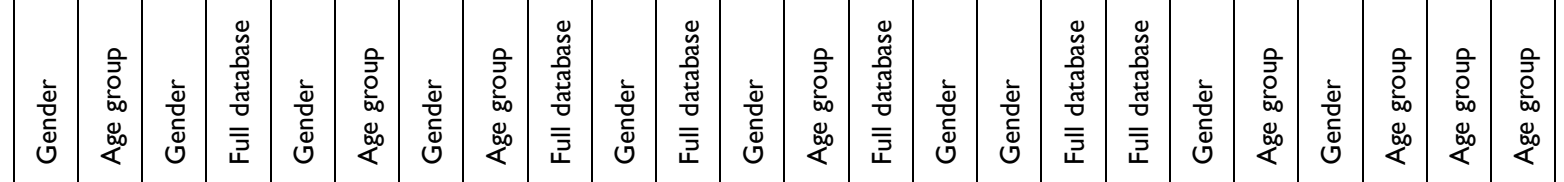

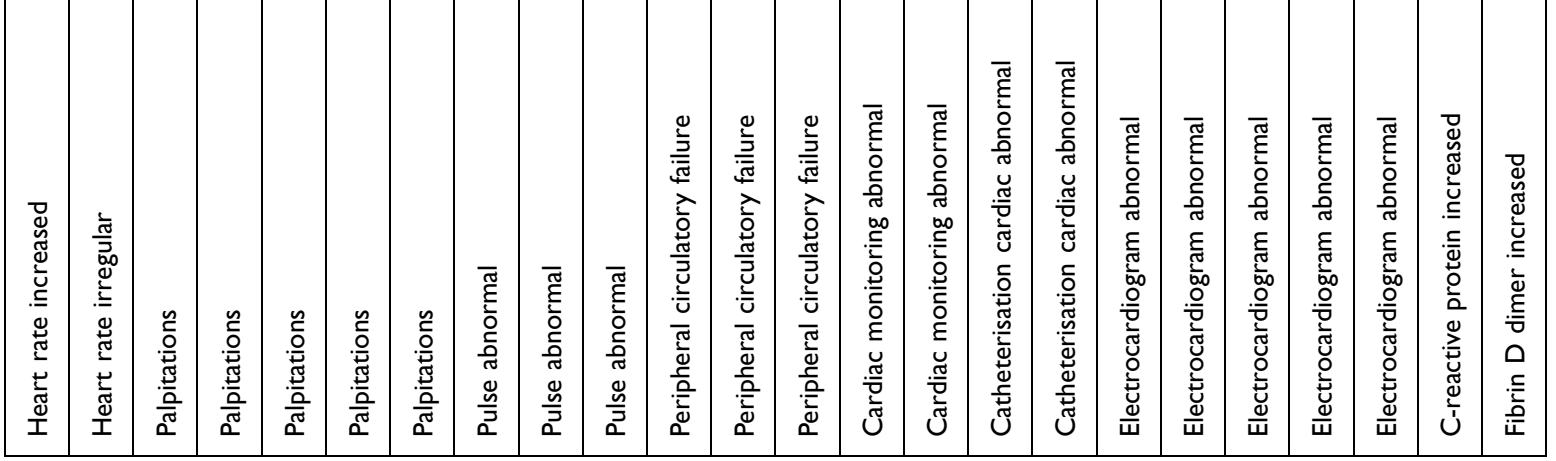




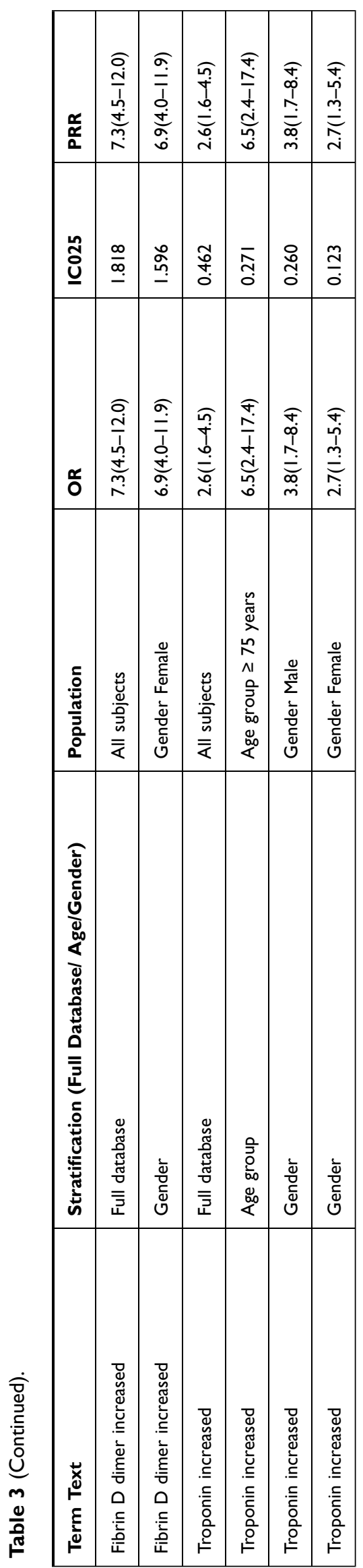

vaccine, but the association of the $\mathrm{AE}$ with the vaccine could not be established and could be a coincidental occurrence. ${ }^{39}$ Even though myocardial infarction events were reported from various trials, the probability was remote.

Hypertension and severe hypertension were associated with vaccine use in almost all age groups and genders. In their Case Series Drug Analysis for their COVID-19 vaccine, AstraZeneca reported 176 events of Hypertension, 96 events of increased blood pressure, three events of systolic hypertension, one event each of diastolic hypertension, hypertensive crisis, hypertensive emergency, hypertensive urgency, and peripheral circulatory failure. ${ }^{34}$ The increased probability can be attributed to the fact that the initial population selected was also associated with comorbidities, and hypertension was one. So, there is a possibility that the increased reported hypertensive AEs were due to the cases that were selected rather than the vaccine itself.

Vaccines were also associated with rhythm disorders like supraventricular tachycardia, sinus tachycardia, paroxysmal tachycardia, palpitations, etc. Peripheral circular failure was also found to be associated with vaccine use. The rhythm disorders as reported by AstraZeneca for their COVID-19 vaccine had 1763 events of palpitations, 622 events of tachycardia, 78 events of atrial fibrillation, 43 events of arrhythmia, 34 events of sinus tachycardia, 21 events of extrasystoles, eight events each of supraventricular tachycardia and tachyarrhythmia and six events of angina unstable. ${ }^{34}$

Amongst the investigations and lab tests, abnormal ECG findings, raised C-reactive protein, increase in D-dimer, increase in troponin was found to be associated with the vaccine used in specific age group or gender or all subjects. AstraZeneca, in the same report, reported that there were 792 events of increased heart rate, 61 events of irregular heart rate, 24 events of decreased heart rate, 16 events of abnormal heart rate, one event of ECG T wave inversion, two events of abnormal ECG, three events of increased $\mathrm{C}$-reactive protein, one event of abnormal C-reactive protein and one event of increased fibrin D-dimer. ${ }^{34}$

Even though many of the CVS AEs were reported following administration, a causality assessment needs to be done to confirm the association because these cardiovascular AEs are usually prevalent in certain age groups. As per the data reported by "Heart Disease and Stroke Statistics-2020 Update: A Report From the American Heart Association", in the age group 20 to 85 years, the risk of development of hypertension in a lifetime was 


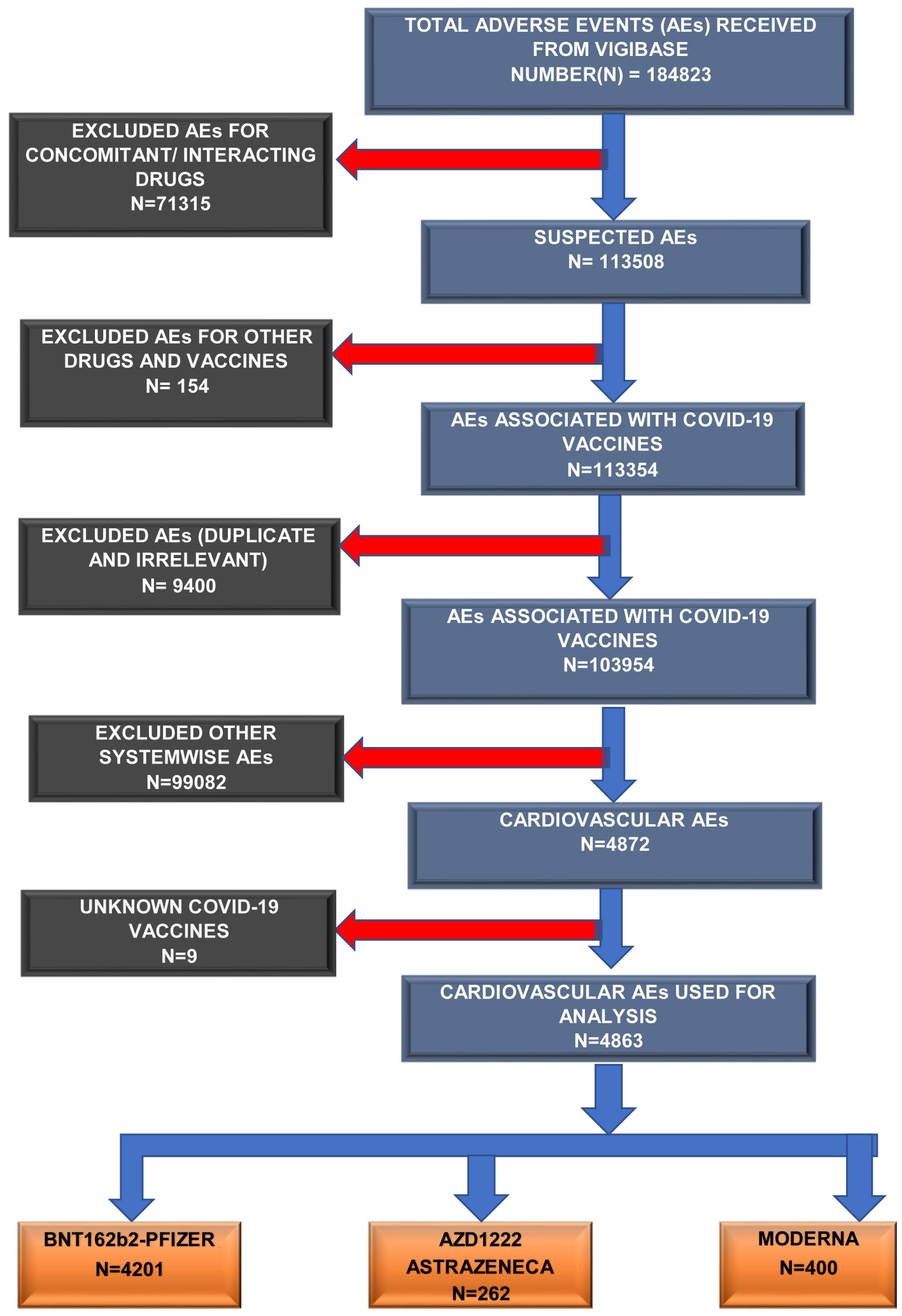

Figure I Schematic diagram of assessment of cardiovascular adverse events associated with COVID-19 vaccines in VigiBase database.

$86.1 \%$ and $85.7 \%$ for black males and females, respectively, and $83.8 \%$ for white males, and $69.3 \%$ for white females, ${ }^{40}$ based on the 2017 National Health Interview
Survey, the age-adjusted prevalence of all types of heart disease was $10.6 \%{ }^{40,41}$ The similar research of "Heart Disease and Stroke Statistics-2021 Update" reported 
that the prevalence of cardiovascular disorder including coronary heart disease, heart failure, hypertension, and stroke in the age group $\geq 20$ years is $49.2 \%$ and on the exclusion of hypertension, the overall cardiovascular risk decreases down to $9.3 \%{ }^{42}$ A study conducted by Zeng et al to study the global prevalence of hypertension reported that it ranged from $13 \%$ to $41 \%$ worldwide. ${ }^{43}$

It has been observed that hypertension is one of the prime factors in patients with atrial fibrillation $(\approx 22 \%){ }^{40,44}$ Study by Khurshid et al reported a prevalence of $2.35 \%$ baseline rhythm abnormality among the general population and a higher (4.84\%) prevalence among population aged 65 to 73 years. ${ }^{45}$ Feinberg et al reported the prevalence of atrial fibrillation to be $2.3 \%$ in individuals more than 40 years and $5.9 \%$ in the population older than 65 years. ${ }^{46}$ Lindberg et al in their study reported a baseline prevalence of $4.9 \%$ among the Swedish population. ${ }^{47}$ It has also been noted that the incidence rate for atrial fibrillation was $31 \%$ higher in the year 2017 as compared to the corresponding incidence in the year $1997 .{ }^{48}$ The prevalence of ischemic heart disease worldwide is about $1.72 \%$ of the world's population. ${ }^{49}$ The tachycardia or cases of increased heart rate can be a physiological response or stress-related response after vaccination, also called Immunization stress-related response (ISRR). ${ }^{50,51}$ The "Coronavirus vaccine -a weekly summary of Yellow Card reporting" research and analysis report observed tachycardia events in patients vaccinated by various COVID-19 vaccines, and the response were attributed as usual was triggered by the body to the vaccines. ${ }^{52}$

The above data of various cardiac disorders among the general population show that the global population is already prone to various cardiovascular disorders like hypertension, coronary artery diseases, rhythm disorders, etc. Hence, the figures reported as adverse events following various COVID-19 vaccination to the VigiBase cannot be considered purely caused by the vaccines themselves. The baseline cardiovascular characteristics of the patients are taken into account to come down to a conclusion. Meylan et al, in their study, reported a case series of nine patients with stage III hypertension after mRNA-Based COVID-19 vaccination; later, it was found that eight out of nine patients had a history of hypertension and were on medication. ${ }^{53}$ As reporting of adverse events considers any events post-administration of a drug or vaccines, causality assessment of all the reported adverse events must be done to conclude. Moreover, time from exposure to adverse events needs to be analyzed to determine whether a particular event is causally associated with the vaccine.

\section{Conclusion}

It is essential to understand that initially, the vaccine was given to old age people and people with comorbidities, and it is expected to notice cardiovascular events like AMI, hypertension, and rhythm disorder in such populations. Hence, the association shown through the disproportionality analysis may not be the true association but expected events in such population. However, the analysis mentioned above may prove to be pathfinding in this regard to prevent further casualties associated with COVID-19. As signals are generated from the analysis, it is essential to review these ADEs to see if there is any causality.

\section{Strength of Study}

This study is based on VigiBase, an extensive database of spontaneous reports of ADEs used by Uppsala Monitoring Centre, Sweden, to generate ADEs related signals and use various published studies to link adverse events with drug use. We used the most conservative disproportionality analysis method to avoid any false-positive association, ie, $\mathrm{IC}_{025}$ values.

\section{Limitations of the Study}

The data in this study were taken from VigiBase, where the information comes from varied sources. The probability of a suspected adverse effect caused by the drug cannot be ascertained in all cases. The information provided does not represent the opinion of the UMC or the WHO.

\section{Data Sharing Statement}

The data that support the findings of this study are available from the corresponding author JC, upon reasonable request.

\section{Consent for Publication}

All authors reviewed and approved the final version and have agreed to be accountable for all aspects of the work, including any issues related to accuracy or integrity.

\section{Author Contributions}

All authors made a significant contribution to the work reported, whether that is in the conception, study design, execution, acquisition of data, analysis, and interpretation, or in all these areas; took part in drafting, revising, or critically reviewing the article; gave final approval of the version to be published; have agreed on the journal to 
which the article has been submitted, and decided to be accountable for all aspects of the work.

\section{Funding}

This paper was not funded.

\section{Disclosure}

The authors declare that they do not have any financial involvement or affiliations with any organization, association, or entity directly or indirectly with the subject matter or materials presented in this article. This also includes honoraria, expert testimony, employment, ownership of stocks or options, patents or grants received or pending, or royalties. The authors are responsible for the views expressed in this paper, and they do not necessarily represent the decisions, policy or views of the World Health Organization.

\section{References}

1. World Health Organisation. WHO coronavirus disease (COVID-19) dashboard; 2021 [cited March 24, 2021]. Available from: https:// covid19.who.int/. Accessed July 16, 2021.

2. Craven J. Regulatory Focus: COVID-19 vaccine tracker: Regulatory Affairs Professionals Society (RAPS); 2021 [updated March 25, 2021; cited March 31, 2021]. Available from: https://www.raps.org/newsand-articles/news-articles/2020/3/covid-19-vaccine-tracker. Accessed July 16, 2021.

3. Polack FP, Thomas SJ, Kitchin N, et al.; C4591001 Clinical Trial Group. Safety and efficacy of the BNT162b2 mRNA COVID-19 vaccine. $N$ Engl $J$ Med. 2020;383(27):2603-2615. doi:10.1056/ NEJMoa2034577.

4. Baden LR, El Sahly HM, Essink B, et al.; COVE Study Group. Efficacy and safety of the mRNA-1273 SARS-CoV-2 Vaccine. $N$ Engl J Med. 2021;384(5):403-416. doi:10.1056/NEJMoa2035389

5. Voysey M, Clemens SAC, Madhi SA, et al.; Oxford COVID Vaccine Trial Group. Safety and efficacy of the ChAdOx $1 \mathrm{nCoV}-19$ vaccine (AZD1222) against SARS-CoV-2: an interim analysis of four randomized controlled trials in Brazil, South Africa, and the UK. Lancet. 2021;397(10269):99-111.

6. European Medicines Agency. COVID-19 Vaccine AstraZeneca: PRAC investigating cases of thromboembolic events - vaccine's benefits currently still outweigh risks - Update; 2021 [updated March 11, 2021; cited March 31, 2021]. Available from: https://www.ema. europa.eu/en/news/covid-19-vaccine-astrazeneca-prac-investigatingcases-thromboembolic-events-vaccines-benefits. Accessed July 16, 2021.

7. Medical News Today. New SARS-CoV-2 variants: how can vaccines be adapted?; 2021 [cited March 31, 2021]. Available from: https:// www.medicalnewstoday.com/articles/new-sars-cov-2-variants-howcan-vaccines-be-adapted. Accessed July 16, 2021.

8. World Health Organisation. The Moderna COVID-19 (mRNA-1273) vaccine: what you need to know; 2021 [updated January 26, 2021; cited March 20, 2021]. Available from: https://www.who.int/news-room/fea ture-stories/detail/the-moderna-covid-19-mrna-1273-vaccine-what-youneed-to-know? gclid=CjwKCAjwxuuCBhATEiwAIIIz0QMTsc3acAzhBS0gcljbpVRGF5zH7tZaPHRg0SXlsj8ZZPWQRODtBo CuogQAvD_BwE. Accessed July 16, 2021.
9. Lee EJ, Cines DB, Gernsheimer T, et al. Thrombocytopenia following pfizer and moderna SARS-CoV-2 vaccination. Am J Hematol. 2021;96(5):534-537. doi:10.1002/ajh.26132

10. Torjesen I. Covid-19: Norway investigates 23 deaths in frail elderly patients after vaccination. $B M J .2021 ; 372: \mathrm{n} 149$.

11. Centers for Disease Control and Prevention. COVID-19 ACIP vaccine recommendations; 2021 [updated March 2, 2021; cited March 22, 2021]. Available from: https://www.cdc.gov/vaccines/hcp/aciprecs/vacc-specific/covid-19.html. Accessed July 16, 2021.

12. Uppsala Monitoring Centre: WHO Programme for International Drug Monitoring. VigiBase; 2020 [cited October 10 2020]. Available from: https://www.who-umc.org/vigibase/vigibase/. Accessed July 16, 2021.

13. Charan J, Kaur RJ, Bhardwaj P, et al. Rapid review of suspected adverse drug events due to remdesivir in the WHO database; findings and implications. Expert Rev Clin Pharmacol. 2021;14(1):95-103. doi:10.1080/17512433.2021.1856655

14. Kaur RJ, Charan J, Dutta S, et al. Favipiravir Use in COVID-19: analysis of suspected adverse drug events reported in the WHO database. Infect Drug Resist. 2020;13:4427-4438. doi:10.2147/IDR. S287934

15. Charan J, Dutta S, Kaur R. et al. Tocilizumab in COVID-19: a study of adverse drug events reported in the WHO database. Expert Opin Drug Saf. 2021;1-12. doi:10.1080/14740338.2021.1946513

16. Dutta S, Kaur RJ, Bhardwaj P, et al. Hydroxychloroquine as therapeutic option in COVID-19: analysis of suspected cardiovascular adverse drug events reported in the vigibase. Bangladesh $j$ Med Sci. 2021;20(4):897-910. doi:10.3329/bjms.v20i4.54150

17. Kaur RJ, Dutta S, Bhardwaj P, et al. Adverse events reported from COVID-19 vaccine trials: a systematic review. Indian $J$ Clin Biochem. 2021;21-23 doi:10.1007/s12291-021-00968-z

18. Glenn DA, Hegde A, Kotzen E, et al. Systematic review of safety and efficacy of COVID-19 vaccines in patients with kidney disease. Kidney Int Rep. 2021;6(5):1407-1410. doi:10.1016/j. ekir.2021.02.011

19. Dutta S, Kaur RJ, Charan J, et al. Serious adverse events reported from the COVID-19 vaccines: a descriptive study based on WHO database. medRxiv. 2021. doi:10.1101/2021.03.23.21253433

20. Uppsala Monitoring Centre: WHO Programme for International Drug Monitoring. VigiBase FAQs; [updated September 16, 2020]. Available from: https://www.who-umc.org/vigibase/vigibase/knowmore-about-vigibase/. Accessed March 7, 2021.

21. Uppsala Monitoring Centre. VigiBase: signaling harm and pointing to safer use. Available from: https://www.who-umc.org/vigibase/vigi base/vigibase-signalling-harm-and-pointing-to-safer-use/. Accessed October 10, 2020.

22. World Health Organization. WHO Collaborating Centre for Drug Statistics Methodology; [updated August 17, 2020]. Available from: https://www.whocc.no/. Accessed April 12, 2021.

23. Brown EG, Wood L, Wood S. The medical dictionary for regulatory activities (MedDRA). Drug Saf. 1999;20(2):109-117. doi:10.2165/ 00002018-199920020-00002

24. International Council for Harmonisation of Technical Requirements for Pharmaceuticals for Human Use (ICH). Medical Dictionary for Regulatory Activities (MedDRA). Available from: https://www.med dra.org/how-to-use/support-documentation/english/welcome. Accessed April 12, 2021.

25. Medical Dictionary for Regulatory Activities (MedDRA). MedDRA Hierarchy. International Council for Harmonisation of Technical Requirements for Pharmaceuticals for Human Use (ICH). Available from: https://www.meddra.org/how-to-use/basics/hierarchy. Accessed April 12, 2021.

26. Uppsala Monitoring Centre. Glossary of pharmacovigilance terms; [updated September 12, 2020]. Available from: https://www.whoumc.org/global-pharmacovigilance/publications/glossary/. Accessed April 12, 2021. 
27. International Council for Harmonisation of Technical Requirements for Pharmaceuticals for Human Use (ICH). Maintenance of the ich guideline on clinical safety data management: data elements for transmission of individual case safety report E2B(R2); March 9, 2021. Available from: https://admin.ich.org/sites/default/files/inlinefiles/E2B_R2_Guideline.pdf. Accessed March 9, 2021.

28. Bate A, Lindquist M, Edwards IR, et al. A Bayesian neural network method for adverse drug reaction signal generation. Eur $J$ Clin Pharmacol. 1998;54(4):315-321. doi:10.1007/s002280050466

29. Montastruc JL, Sommet A, Bagheri H, Lapeyre-Mestre M. Benefits and strengths of the disproportionality analysis for identification of adverse drug reactions in a pharmacovigilance database. Br J Clin Pharmacol. 2011;72(6):905-908. doi:10.1111/j.13652125.2011.04037.x

30. Li C, Xia J, Deng J, et al. A comparison of measures of disproportionality for signal detection on adverse drug reaction spontaneous reporting database of Guangdong province in China. Pharmacoepidemiol Drug Saf. 2008;17(6):593-600. doi:10.1002/ pds. 1601

31. Harpaz R, DuMouchel W, Shah NH, et al. Novel data-mining methodologies for adverse drug event discovery and analysis. Clin Pharmacol Ther. 2012;91(6):1010-1021. doi:10.1038/clpt.2012.50

32. Bate A, Evans SJ. Quantitative signal detection using spontaneous ADR reporting. Pharmacoepidemiol Drug Saf. 2009;18(6):427-436. doi:10.1002/pds. 1742

33. Meo SA, Bukhari IA, Akram J, Meo AS, Klonoff DC. COVID-19 vaccines: comparison of biological, pharmacological characteristics and adverse effects of Pfizer/BioNTech and moderna vaccines. Eur Rev Med Pharmacol Sci. 2021;25(3):1663-1669.

34. AstraZeneca. COVID-19 vaccine AstraZeneca analysis print; May 30, 2021 [updated May 20, 2021]. https://assets.publishing.service. gov.uk/government/uploads/system/uploads/attachment_data/file/ 972833/COVID-19_AstraZeneca_Vaccine_Analysis_Print.pdf. Accessed May 30, 2021.

35. FDA Briefing Document: Moderna COVID-19 Vaccine. Vaccines and related biological products advisory committee meeting; [updated December 17, 2020]. https://www.fda.gov/media/144434/download. Accessed March 9, 2021.

36. FDA Briefing Document: Pfizer-BioNTech COVID-19 Vaccine. Vaccines and related biological products advisory committee meeting; [updated March 8, 2021]. Available from: https://www.fda.gov/ media/144245/download. Accessed July 16, 2021. Accessed March $18,2021$.

37. Publishing Service. Government of UK. COVID-19 mRNA PfizerBioNTech Vaccine Analysis Print. Updated May 28, 2021. Available from: https://assets.publishing.service.gov.uk/government/uploads/ system/uploads/attachment_data/file/989996/COVID-19_mRNA_ Pfizer-_BioNTech_Vaccine_Analysis_Print.pdf. Accessed June 4, 2021.

38. Shimabukuro T Centers for Disease Control and Prevention. COVID19 vaccine safety update: Advisory Committee On Immunization Practices (ACIP) January 27, 2021. CDC COVID-19 Vaccine Task Force; March 30, 2021. [updated January 27, 2021]. Available from: https://www.cdc.gov/vaccines/acip/meetings/downloads/slides-202101/06-COVID-Shimabukuro.pdf. Accessed March 30, 2021.

39. Boivin Z. Untimely myocardial infarction or COVID-19 vaccine side effect. Cureus. 2021;13(3):e13651. doi:10.7759/cureus.13651
40. Virani SS, Alonso A, Benjamin EJ, et al. Heart disease and stroke statistics-2020 update: a report from the American Heart Association. Circulation. 2020;141(9):e139-e596. doi:10.1161/ cir.0000000000000757

41. Villarroel MA, Blackwell DL, Jen A. Tables of Summary Health Statistics for U.S. Adults: 2018 National Health Interview Survey. NCHS, National Center for Health Statistics. 2019. Available from: http://www.cdc.gov/nchs/nhis/SHS/tables.htm. Accessed May 29, 2021.

42. Virani SS, Alonso A, Aparicio HJ, et al. Heart disease, and stroke statistics-2021 update: a report from the American Heart Association. Circulation. 2021;143(8):e254-e743. doi:10.1161/ cir.00000000000000950

43. Zeng Z, Chen J, Xiao C, Chen W. A global view on prevalence of hypertension and human develop index. Ann Glob Health. 2020;86 (1):67. doi:10.5334/aogh.2591

44. Huxley RR, Lopez FL, Folsom AR, et al. Absolute and attributable risks of atrial fibrillation in relation to optimal and borderline risk factors: the Atherosclerosis Risk in Communities (ARIC) study. Circulation. 2011;123(14):1501-1508. doi:10.1161/ circulationaha.110.009035

45. Khurshid S, Choi SH, Weng LC, et al. Frequency of cardiac rhythm abnormalities in a half million adults. Circ Arrhythm Electrophysiol. 2018;11(7);e006273. doi:10.1161/circep.118.006273

46. Feinberg WM, Blackshear JL, Laupacis A, Kronmal R, Hart RG. Prevalence, age distribution, and gender of patients with atrial fibrillation: analysis and implications. Arch Intern Med. 1995;155(5):469473. doi:10.1001/archinte. 1995.00430050045005

47. Lindberg T, Wimo A, Elmståhl S, Qiu C, Bohman DM, Sanmartin Berglund J. Prevalence and incidence of atrial fibrillation and other arrhythmias in the general older population: findings from the Swedish National Study on aging and care. Gerontol Geriatr Med. 2019;5:2333721419859687. doi:10.1177/2333721419859687

48. Lippi G, Sanchis-Gomar F, Cervellin G. Global epidemiology of atrial fibrillation: an increasing epidemic and public health challenge. Int J Stroke. 2021;16(2):217-221. doi:10.1177/1747493019897870

49. Khan MA, Hashim MJ, Mustafa H, et al. Global epidemiology of Ischemic heart disease: results from the Global Burden of Disease Study. Cureus. 2020;12(7):e9349. doi:10.7759/cureus.9349

50. Quer G, Gadaleta M, Radin JM, et al. The physiologic response to COVID-19 vaccination. he physiologic response to COVID-19 vaccination. medRxiv [Preprint]. 2021. doi:10.1101/ 2021.05.03.21256482

51. World Health Organization. Immunization stress-related responses. A Manual.Available from: https://www.who.int/publications/i/item/97892-4-151594-8. Accessed June 4, 2021.

52. Medicines and Healthcare products Regulatory Agency. Coronavirus vaccine - weekly summary of Yellow Card reporting; [updated Jun 3, 2021]. Available from: https://www.gov.uk/government/publications/ coronavirus-covid-19-vaccine-adverse-reactions/coronavirus-vac cine-summary-of-yellow-card-reporting. Accessed June 4, 2021.

53. Meylan S, Livio F, Foerster M, Genoud PJ, Marguet F, Wuerzner G. Stage III hypertension in patients after mRNA-based SARS-CoV-2 Vaccination. Hypertension. 2021;77(6):e56-e57. doi:10.1161/ HYPERTENSIONAHA.121.17316 


\section{Publish your work in this journal}

The International Journal of General Medicine is an international, peer-reviewed open-access journal that focuses on general and internal medicine, pathogenesis, epidemiology, diagnosis, monitoring and treatment protocols. The journal is characterized by the rapid reporting of reviews, original research and clinical studie across all disease areas. The manuscript management system is completely online and includes a very quick and fair peer-review system, which is all easy to use. Visit http://www.dovepress.com/ testimonials.php to read real quotes from published authors.

Submit your manuscript here: https://www.dovepress.com/international-journal-of-general-medicine-journal 